\title{
Direct calculation of the crystal-melt interfacial free energies for continuous potentials: Application to the Lennard-Jones system
}

\author{
Ruslan L. Davidchack \\ Department of Mathematics and Computer Science, University of Leicester, \\ Leicester LE1 7RH, United Kingdom \\ Brian B. Laird \\ Department of Chemistry, University of Kansas, Lawrence, Kansas 66045
}

(Received 16 December 2002; accepted 31 January 2003)

\begin{abstract}
Extending to continuous potentials a cleaving wall molecular dynamics simulation method recently developed for the hard-sphere system [Phys. Rev. Lett. 85, 4751 (2000)], we calculate the crystalmelt interfacial free energies, $\gamma$, for a Lennard-Jones system as functions of both crystal orientation and temperature. At the triple point, $T^{*}=0.617$, the results are consistent with an earlier cleaving potential calculation by Broughton and Gilmer [J. Chem. Phys. 84, 5759 (1986)], however, the greater precision of the current calculation allows us to accurately determine the anisotropy of $\gamma$. From our data we find that, at all temperatures studied, $\gamma_{111}<\gamma_{110}<\gamma_{100}$. A comparison is made to the results from our previous hard-sphere calculation and to recent results for Ni by Asta, Hoyt, and Karma [Phys. Rev. B 66 100101(R) (2002)]. (C) 2003 American Institute of Physics.

[DOI: 10.1063/1.1563248]
\end{abstract}

\section{INTRODUCTION}

The magnitude and orientational dependence (anisotropy) of the solid-liquid interfacial free energy, $\gamma$, is a primary controlling parameter in the kinetics and morphology of crystal growth from the melt, ${ }^{1}$ especially in the case of dendritic growth. ${ }^{2}$ As a consequence, the ability to accurately measure or predict this quantity for specific materials is of significant technological and scientific importance. For most materials, the only experimental data for $\gamma$ is extracted indirectly from nucleation data (assuming some level of classical nucleation theory). ${ }^{1,3,4}$ Such indirect measurements tend to underestimate the actual interfacial free energy by $10 \%-20 \%$ and represent orientational averages, so all information as to interfacial anisotropy is lost. Direct experimental measurements, usually involving contact angle studies, are quite difficult and relatively few in number, ${ }^{5}$ and, with the exception of a small number of studies on transparent organic materials, ${ }^{6,7}$ are not of sufficient precision to resolve anisotropy. This paucity of reliable direct experimental measurements on technologically useful materials (such as metals) has motivated the development of a variety of novel computational methods to determine $\gamma$ via molecular simulation. ${ }^{8-11}$

The interfacial free energy of a crystal-melt interface is defined $^{1}$ as the reversible work required to form a unit area of interface. In a simulation this can be accomplished by constructing a continuous thermodynamic path from an initial system consisting of separated bulk crystal and liquid to a final state containing an interface. The value of $\gamma$ is then determined by thermodynamic integration ${ }^{12}$ along that path. This is a tedious process and care must to be taken to ensure that the process is reversible, i.e., integration along the path in both the forward and backward directions yields the same result (no hysteresis). The first such calculation on a crystal- melt interface was performed by Broughton and Gilmer ${ }^{8}$ on a system of particles interacting with a Lennard-Jones (LJ) potential (truncated so that both the potential and the force vanish at $2.5 \sigma$, where $\sigma$ is the usual LJ diameter). To perform the thermodynamic integration they employed external cleaving potentials that were slowly turned on to separate the samples. The precise (rather complex) functional forms of the cleaving potentials were chosen, more or less by trial and error, to minimize hysteresis. The values of $\gamma$ were determined to be $0.35(2), 0.34(2)$, and $0.36(2)$ (in units of $\epsilon / \sigma^{2}$ ) for the [111], [100], and [110] crystal orientations, respectively. The numbers in parentheses give the uncertainties in the last digit shown. The main source of this error is the small amount of hysteresis in the cleaving of the liquid phase. Unfortunately, the precision of these results was not sufficient to resolve the anisotropy of the interfacial free energy for this system.

Recently, we have developed a modification of the Broughton and Gilmer approach in which planar cleaving walls, as opposed to cleaving potentials, are used to separate the phases. These walls are constructed out of the same type of particles as present in the system, with a two-dimensional (2-D) geometry consistent with the symmetry of the interfacial orientation under study. This method was originally applied to the crystal-melt interface of a system of hard spheres $^{9}$ and was shown to have sufficient precision to resolve the anisotropy with [111] being the lowest free energy face, followed by [100] and [110]. The cleaving wall method is complementary to a method due to Hoyt, Asta, and $\mathrm{Karma}^{10}$ in which the interfacial free energy is extracted from the interfacial stiffness, which is determined from the spectrum of fluctuations in an interfacial position. The cleaving wall method has an advantage in that it requires simulations with an order of magnitude fewer particles than those 
required for the fluctuation method $\left(\mathcal{O}\left[10^{4}\right]\right.$ versus $\left.\mathcal{O}\left[10^{5}\right]\right)$. The precision in the raw values of the interfacial free energies is greater for the cleaving wall method than in the fluctuation approach (even considering the far smaller system samples); however, the fluctuation method yields somewhat more precise values of the anisotropy parameters since these are obtained from the anisotropy of the interfacial stiffness, which is far more anisotropic than the interfacial free energy.

In this work, we extend our cleaving wall approach to systems of particles interacting with continuous potentials, specifically applying it to the system of truncated LJ particles considered by Broughton and Gilmer. Our results at the triple point are consistent with the Broughton and Gilmer calculation, but are of greater precision, allowing us to resolve the anisotropy, which is found to differ slightly from that for the hard-sphere system. ${ }^{9}$ In addition, we determine the temperature dependence of $\gamma$ along the coexistence curve. The magnitude of $\gamma$ is shown to scale roughly linearly with the melting temperature (as predicted by the hardsphere model). ${ }^{13}$

\section{THE CLEAVING WALL METHOD}

The direct determination of the excess free energy of the crystal-fluid interface of a model system within a computer simulation can be achieved by thermodynamic integration along a reversible path beginning with separate crystal and fluid bulk systems prepared at the coexistence conditions and ending with a system containing a crystal-liquid interface at equilibrium with the surrounding bulk phases. The construction of such a path requires the development of a procedure to reversibly cleave a simulation box into two noninteracting systems. Following the prescription of Broughton and Gilmer, ${ }^{8}$ we identify the following steps in the process of creating the crystal-liquid interface (see Fig. 1).

(1) Step 1: Split the crystal bulk system with a suitably chosen "cleaving" potential while maintaining the periodic boundary conditions.

(2) Step 2: Split the liquid system in a similar way.

(3) Step 3: Juxtapose the cleaved crystal and liquid systems by rearranging the boundary conditions while maintaining the cleaving potentials.

(4) Step 4: Remove the cleaving potentials from the combined system.

The interfacial free energy is calculated as the total work required to perform the above steps divided by the area of the constructed interface. In addition to the coexistence conditions, the result will also depend on the orientation of the crystal with respect to the interfacial plane. We will refer to the plane along which the crystal and liquid systems are split as the cleaving plane. The location of the cleaving plane in the crystal system is chosen in the center of the simulation box between two crystal layers, while in the liquid system the precise location is arbitrary.

The reversibility and precision of the thermodynamic integration process are very sensitive to the choice of cleaving potentials. The requirements for the cleaving potential are two-fold: First, the cleaving potential should perturb the sys-
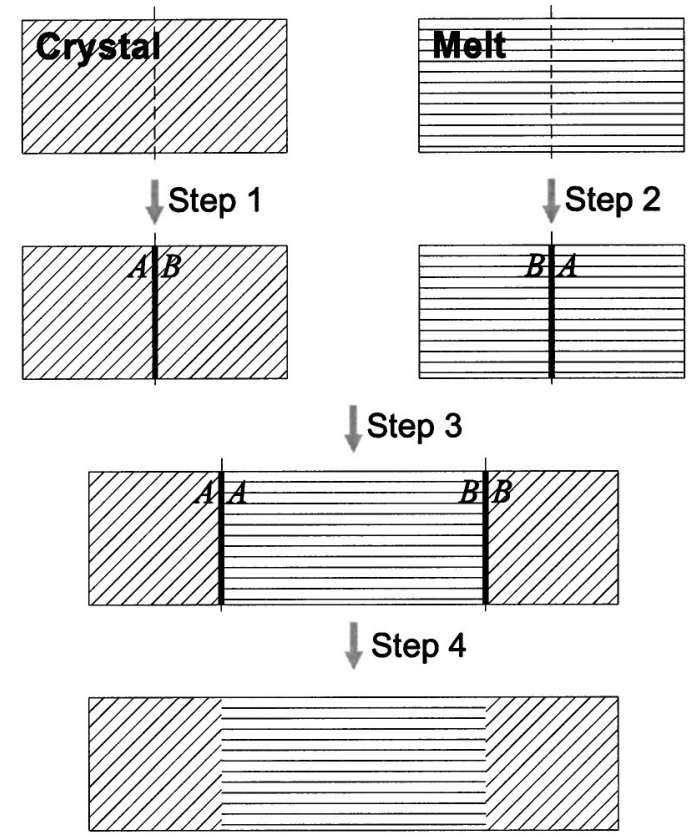

FIG. 1. An illustration of the four-step reversible process of creating the crystal-liquid interface from separate bulk systems. Dashed lines show the location of the cleaving planes and thick solid lines represent the cleaving potential. Letters $A$ and $B$ label the crystal and liquid on either side of the cleaving planes for a better illustration of the boundary conditions rearrangement in step 3. Periodic boundary conditions are assumed on all boundaries of the simulation boxes.

tem as little as possible. As a consequence, it is desirable that, in step 2, the potential introduces structure into the cleaved liquid that is compatible with the structure of the crystal layers. Second, the cleaving potential must be strong enough to prevent the particles from crossing the cleaving plane. Otherwise, the rearrangement of the boundary conditions in step 3 cannot be performed.

Broughton and Gilmer ${ }^{14}$ carefully designed a set of cleaving potentials for the truncated LJ system. However, their approach for constructing the cleaving potential was optimized specifically for the LJ system and is not easily adaptable to a general case. Here we outline an approach that is easily adaptable to systems with different interparticle interaction potentials and different crystal structures.

As was demonstrated in our recent calculation of the interfacial free energy for the hard-sphere system, ${ }^{9}$ the appropriate structure in the interfacial region of the fluid can be easily introduced by the potential of a pair of "cleaving walls" (labeled 1 and 2), made of properly oriented crystal layers, each interacting only with the particles on the opposite side of the cleaving plane. The wall particles are held fixed at the crystal lattice sites. When the two walls are far away from the cleaving plane, they do not interact with the system. Moving the walls closer to the cleaving plane introduces a cleaving potential whose structure is similar to that outside the crystal layers of the studied system. When a liquid system interacts with such a cleaving wall, it is expected to form an interfacial layer structure similar to that at the crystal-liquid interface. To achieve this, the interaction po- 
tential of the wall particles must be similar to that of the system particles.

Since interactions in the crystal are usually dominated by the short-range repulsive part of the potential, it is sufficient to choose the interaction potential of the wall particles as a monotonically decreasing function $\phi(r)$ with a relatively small cut-off radius $r_{w}$, which models the repulsive core of the interparticle potential in the system under investigation. An interaction of the system with each cleaving wall is then given by

$$
\begin{aligned}
& \Phi_{1}(\mathbf{r} ; z)=\sum_{j} \phi\left(\left|\mathbf{r}-\mathbf{R}_{j}^{(1)}+\mathbf{n} z\right|\right), \\
& \Phi_{2}(\mathbf{r} ; z)=\sum_{j} \phi\left(\left|\mathbf{r}-\mathbf{R}_{j}^{(2)}-\mathbf{n} z\right|\right),
\end{aligned}
$$

where $\mathbf{R}_{j}^{(1,2)}$ are the positions of the particles forming the walls, $\mathbf{n}$ is a unit vector normal to the cleaving plane, and $z$ measures the distance of the walls to the cleaving plane.

Next, we need to ensure that the system on the one side of the cleaving plane interacts only with the wall on the other side of the plane. To achieve this, we use the monotonic character of the potential $\phi(r)$ and define the cleaving potential as the minimum of the two wall potentials, namely,

$$
\Phi(\mathbf{r} ; z)=\min \left(\Phi_{1}, \Phi_{2}\right),
$$

which decays to zero away from the cleaving plane, as long as the potential $\phi(r)$ is chosen to be a positive monotonically decreasing function. It is a simple exercise to show that the vector arguments defined in Eq. (1) combined with the minimum function defined above guarantees that each particle interacts with the proper wall. To remove discontinuity of the gradient of $\Phi$ at the points where $\Phi_{1}=\Phi_{2}$, we slightly modify the minimum function as follows:

$$
m(x, y)=\left\{\begin{array}{l}
x, \quad x \leqslant y-\delta(x+y), \\
y, \quad x \geqslant y+\delta(x+y), \\
p(x, y), \quad \text { otherwise, }
\end{array}\right.
$$

with

$$
p(x, y)=\frac{x+y}{4}(2-\delta)-\frac{(x-y)^{2}}{4 \delta(x+y)},
$$

and parameter $\delta$ characterizing the relative width of the interpolation region. We set $\delta=0.25$ for the present study. The cleaving potential is defined as

$$
\Phi(\mathbf{r} ; z)=m\left(\Phi_{1}, \Phi_{2}\right) .
$$

Even though the crystal system does not require additional ordering and, as proposed by Broughton and Gilmer, can be cleaved with a short-range repulsive potential centered at the cleaving plane, we have found that using the same cleaving potential for both crystal and liquid systems minimizes the error during the thermodynamic integration in step 3 .
To calculate the reversible work in steps 1,2 , and 4 , we can use the wall position $z$ as the integration coordinate. The reversible work is thus determined by evaluating the integral,

$$
w_{1,2,4}=-\int_{z_{i}}^{z_{f}}\left\langle\frac{\partial \Phi}{\partial z}\right\rangle d z
$$

where the angle brackets denote averaging over a simulation run at a fixed cleaving wall position. In steps 1 and 2 , the initial position of the cleaving walls, $z_{i}$, is just outside the range of the interaction potential determined by the cut-off radius $r_{w}$. The final wall position $z_{f}$ is determined by the requirement that the cleaving potential is sufficiently strong to prevent the particles from crossing the cleaving plane. In step 4, the initial and final positions of the walls are reversed. Because of the repulsive character of the cleaving potential, the work in steps 1 and 2 is expected to be positive, while in step 4 it is negative.

In step 3, the boundary conditions are gradually rearranged using a coupling parameter $\lambda$. The total interaction energy in step 3 is given by

$$
\begin{aligned}
U(\lambda)= & (1-\lambda) \sum_{i<j, A B} u\left(r_{i j}\right)+\lambda \sum_{i<j, A A} u\left(r_{i j}\right) \\
& +\sum_{i} \Phi\left(\mathbf{r}_{i} ; z_{f}\right)
\end{aligned}
$$

where the letters $(A B)$ refer to the boundary conditions with crystal-crystal and liquid-liquid interactions across the cleaving planes, and $(A A)$ refer to the boundary conditions with crystal-liquid interactions (see the diagram). The last term represents the cleaving potential at the final wall positions in steps 1 and 2. The work done during step 3 is determined from the integral

$$
w_{3}=\int_{0}^{1}\left\langle\frac{\partial U}{\partial \lambda}\right\rangle d \lambda \text {, }
$$

where

$$
\frac{\partial U}{\partial \lambda}=\sum_{i<j, A A} u\left(r_{i j}\right)-\sum_{i<j, A B} u\left(r_{i j}\right) .
$$

Note that this procedure depends upon the interface being flat on average; i.e., the interface is generally "rough" on a short time scale, but the fluctuations in the interfacial position should average to a nearly planar surface. With the preparation procedure described above, this condition is easily satisfied for the system sizes used.

\section{RESULTS FOR TRUNCATED LENNARD-JONES POTENTIAL}

In order to have direct comparison with the results of Broughton and Gilmer, ${ }^{14}$ we have used the same modification of the Lennard-Jones potential, namely ${ }^{15}$ 


$$
u_{t \mathrm{LJ}}(r)=\left\{\begin{array}{l}
4 \epsilon\left[\left(\frac{\sigma}{r}\right)^{12}-\left(\frac{\sigma}{r}\right)^{6}\right]+C_{1}, \quad r \leqslant 2.3 \sigma, \\
C_{2}\left(\frac{\sigma}{r}\right)^{12}+C_{3}\left(\frac{\sigma}{r}\right)^{6}+C_{4}\left(\frac{r}{\sigma}\right)^{2}+C_{5}, \quad 2.3 \sigma<r<2.5 \sigma, \\
0, \quad 2.5 \sigma \leqslant r,
\end{array}\right.
$$

where $C_{1}=0.016132 \epsilon, C_{2}=3136.6 \epsilon, C_{3}=-68.069 \epsilon, C_{4}$ $=-0.083312 \epsilon$, and $C_{5}=0.74689 \epsilon$. (Note: the sign of $C_{4}$ was incorrectly reported as positive in the Broughton and Gilmer original publication.) This potential has a continuous first derivative and a small discontinuity in its second derivative at $r=2.3 \sigma$.

To achieve optimal performance of the simulation at all stages of the cleaving process, we use a cell-assisted force evaluation method. ${ }^{16}$ The simulation region is subdivided into cells with edge sizes just exceeding the interatomic interaction range. When atoms are assigned to cells according to their current position, only interactions between atoms in the same cell or in immediately adjacent cells needs to be considered. Such a method is particularly useful in step 3, where interaction forces must be computed for two different boundary conditions [see Eq. (7)]. With the cell-assisted method the additional computational effort is limited to the cell layers immediately adjacent to the cleaving planes. (Note: the cell subdivision is carried out in such a way that the cell boundary coincides with the cleaving plane.)

Since the insertion of the exteral cleaving potentials ("walls") breaks the translational symmetry of the system Hamiltonian, and linear momentum is no longer strictly conserved. This leads to a problem for long runs in that the crystal, taken as a whole, can drift relative to the cleaving plane. To prevent this, we immobilize the innermost two crystal layers by assigning them an infinite mass. In the datacollection runs these fixed planes were about 20 crystal layers away from the nearest crystal-melt interface, and should not have a significant effect on the interfacial properties. To check this, we have performed smaller simulations with an identical cross-sectional area, but fewer crystal layers (where the interface is about $12-14$ crystal layers distant from the fixed planes) and obtain results identical, within simulation error, to the larger systems.

For the present study, we calculate $\gamma$ at both the triple point temperature of $T^{*} \equiv k_{\mathrm{B}} T / \epsilon=0.617$ (as determined by Broughton and Gilmer ${ }^{17}$ ), and at higher temperatures $\left(k_{\mathrm{B}} T / \epsilon=1.0\right.$ and 1.5$)$. The crystal-liquid coexistence parameters at these temperatures are summarized in Table I. [Note the slightly negative pressure at $k_{\mathrm{B}} T / \epsilon=0.617$. We have

TABLE I. Coexistence conditions for the modified Lennard-Jones potential of Eq. (10).

\begin{tabular}{lccc}
\hline \hline$T, k_{\mathrm{B}} \epsilon^{-1}$ & $\rho_{c}, \sigma^{-3}$ & $\rho_{l}, \sigma^{-3}$ & $P, \epsilon \sigma^{-3}$ \\
\hline 0.617 & 0.945 & 0.828 & -0.02 \\
1.0 & 1.005 & 0.923 & 4.95 \\
1.5 & 1.074 & 1.003 & 12.9 \\
\hline \hline
\end{tabular}

found that $k_{\mathrm{B}} T / \epsilon=0.618$ is a better estimate of the triple point temperature for the modified LJ potential of Eq. (10). However, to have a direct comparison with the results of Ref. 17, we have calculated interfacial free energy at the lower temperature.] The parameters are obtained by monitoring long simulation runs of the crystal-liquid interfacial systems. If the initial conditions are shifted from those at coexistence, such a system equilibrates itself through melting/ freezing at the interfaces. During the runs, the pressure tensor profiles ${ }^{18}$ are monitored and the simulation box rescaled, if necessary, to remove any stress in the bulk crystal. The equations of motion are integrated using the velocityVerlet algorithm ${ }^{19}$ with the step sizes $\Delta t=0.005,0.004$, and 0.003 [in units of $\left(m \sigma^{2} / \epsilon\right)^{1 / 2}$, where $m$ is the particle mass], for the simulations at temperatures $T^{*}=0.617,1.0$, and 1.5, respectively.

At each of the three temperatures, we calculate the interfacial free energy for three crystal orientations: [100], [110], and [111]. For step 1, the crystal system is prepared by placing particles in the ideal crystal configuration with random initial velocities and running the simulation for 5000 time steps, rescaling the velocities every 1000 steps to reach the equilibrium state at the specified temperature. The scaling factor is determined from the value of the average kinetic energy per particle during the preceding 1000 steps. The size $\left(L_{x}, L_{y}, L_{z}\right)$ and number of particles $\left(N_{c}\right)$ for systems with different orientations are as follows:

- [100]: $L_{x}=L_{y}=9 a, L_{z}=20 a, \quad N_{c}=6480$;

- [110]: $L_{x}=6 \sqrt{2} a \approx 8.5 a, L_{y}=9 a$, $L_{z}=12 \sqrt{2} a \approx 17 a, \quad N_{c}=5184$

- [111]: $L_{x}=5.5 \sqrt{2} a \approx 7.8 a, \quad L_{y}=3 \sqrt{6} a \approx=7.3 a$, $L_{z}=12 \sqrt{3} a \approx 20.8 a, \quad N_{c}=4752 ;$

where $a=\left(4 / \rho_{c}\right)^{1 / 3}$ is the size of the fcc unit cell, approximately equal to $1.618 \sigma, 1.585 \sigma$, and $1.550 \sigma$, for the temperatures $T^{*}=0.617,1.0$, and 1.5 , respectively. These sizes correspond to 162,108 , and 132 particles per crystal layer for the [100], [110], and [111] interfaces, respectively. The total area of the crystal-liquid interface that is formed after step 4 is equal to $A=2 L_{x} L_{y}$.

To form the liquid system for step 2, we start with an ideal crystal block of exactly the same size and number of particles as in step 1, and then remove particles at random to reduce the density from $\rho_{c}$ to $\rho_{l}$. Thus, the number of particles in the liquid system is equal to the nearest integer of $N_{c} \rho_{l} / \rho_{c}$. The equilibration run of 10000 steps is then performed, which is sufficient to reach the equilibrium liquid state with the given temperature and density. The velocities are scaled in the same manner as in step 1. 


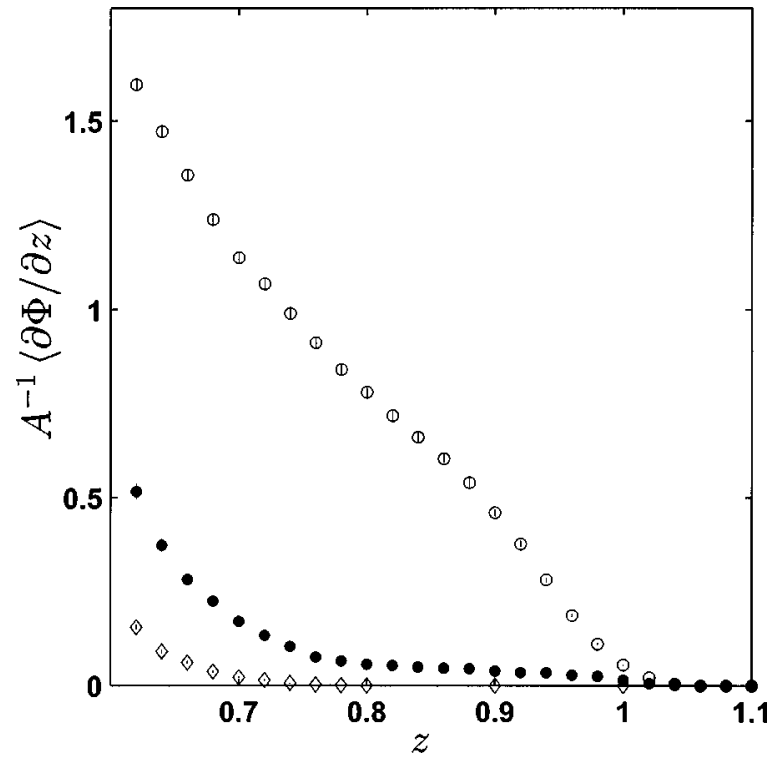

FIG. 2. Integrand for thermodynamic integration in steps 1 (diamonds), 2 (open circles), and 4 (solid dots) for the [111] interface at $T^{*}=0.617$. The error bars, indicated by vertical lines over each symbol, are two standard deviations.

Next the cleaving walls are introduced into the crystal and liquid systems. The cleaving wall is constructed from a single crystal layer for the [100] and [111] orientations and from two layers for the [110] orientation (this was necessary to prevent atoms from crossing through this non-closepacked face). As discussed in the previous section, the cleaving potential $\phi(r)$ is constructed from the repulsive core of the LJ potential, which we define using a standard Weeks-Chandler-Anderson ${ }^{23}$ splitting:

$$
\phi(r)=\left\{\begin{array}{l}
4 \epsilon\left[\left(\frac{\sigma}{r}\right)^{12}-\left(\frac{\sigma}{r}\right)^{6}\right]+\epsilon, \quad r<r_{w}=2^{1 / 6} \sigma, \\
0, \quad r_{w} \leqslant r .
\end{array}\right.
$$

In steps 1 and 2 the cleaving potential is gradually introduced by changing the value of $z$ in Eq. (5) from $z_{i}$ to $z_{f}$, typically in increments of $0.02 \sigma$ (see Fig. 2), with the initial system state for the new value of $z$ being the final state from the previous $z$ value. In our simulations, the initial $z$ value, $z_{i}$ is $1.1 \sigma$ (which is large enough to ensure that the walls do not interact with any particles in the system so that the insertion work is zero), and the final value, $z_{f}$, varies from 0.50 to 0.64 depending upon the orientation and temperature of the interfacial system - this is chosen to be large enough that the two sides are permanently separated. At each $z$ an equilibration run of 3000 steps is followed by 25000 steps used to calculate the average value and statistical error of $\partial \Phi / \partial z$. As in the initial equilibration runs, the velocities are rescaled every 1000 steps to retain a constant value of the temperature. For an illustration of the method we show in Fig. 2 the thermodynamic integration integrand for steps 1,2 , and 4 for the [111] interface at $T^{*}=0.617$ (using $z_{f}=0.62$ ). For that same interface and temperature, the integrand for step 3 is shown in Fig. 3 with an inset highlighting the region of maximum hysteresis error. The integrals in Eqs. (6) and (8)

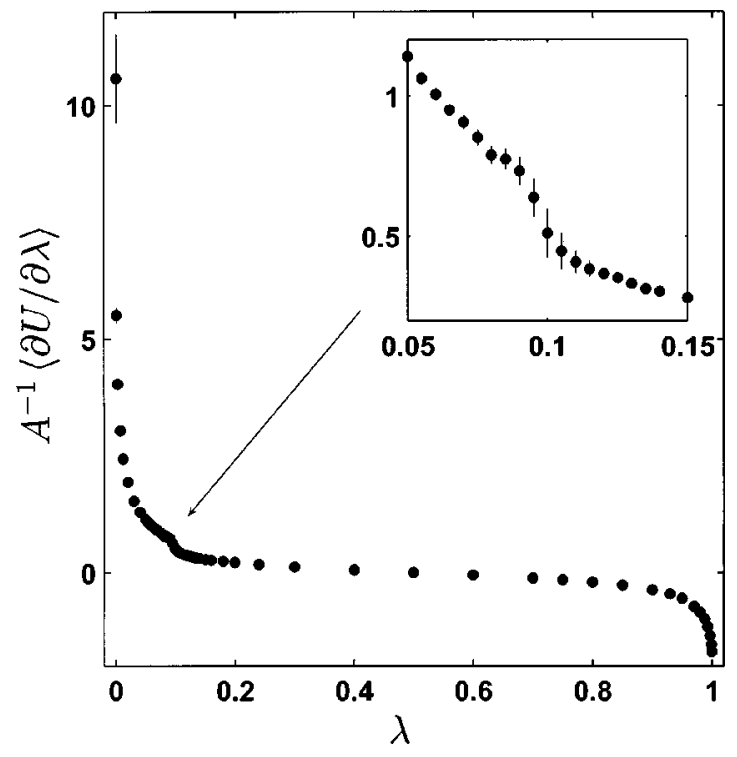

FIG. 3. Integrand for thermodynamic integration in step 3 for the [111] interface at $T^{*}=0.617$. The inset shows a magnification of the region primarily affected by hysteresis, reflected by the larger than average error bars.

are calculated using the trapezoidal rule. The results are summarized in Table II. To test system size effects, we have repeated the calculations for systems that are about half the interfacial area and a third shorter (e.g., $6 a \times 6 a \times 12 a$ for [100]). The values of the integrands for the smaller systems were within the error bars of those for the larger systems.

Even though the relative statistical accuracy that can be achieved for a given duration of the simulation run is about the same for all temperatures and orientations (approximately $0.5 \%$ ), we see that the relative error range in Table II increases with the temperature, especially for the [111] orientation. This increase is due to the observation that reversibility (as measured by the lack of hysteresis) of the thermodynamic integration process for the LJ system is more difficult to achieve for higher temperatures.

There are two sources of potential hysteresis in the fourstep process of creating an interface. The first one is associated with the liquid ordering transition that occurs either at the end of step 2, or at the beginning of step 3. For lower temperatures, the hysteresis can be essentially eliminated by increasing the duration of the equilibration runs near the point of transition. However, the hysteresis for higher temperatures is more persistent, especially in the case of the [111] orientation. This may be due to a particular choice of the cleaving potential. More research is necessary to elucidate the origin of the hysteresis and ways to eliminate it.

TABLE II. Interfacial free energy (in units of $\epsilon \sigma^{-2}$ ) for the truncated Lennard-Jones potential for selected temperatures and crystal orientations. Numbers in parentheses indicate the estimated error on the last digit(s) shown.

\begin{tabular}{lccc}
\hline \hline & $T^{*}=0.617$ & 1.0 & 1.5 \\
\hline$\gamma_{100}$ & $0.371(3)$ & $0.562(6)$ & $0.84(2)$ \\
$\gamma_{110}$ & $0.360(3)$ & $0.543(6)$ & $0.82(2)$ \\
$\gamma_{111}$ & $0.347(3)$ & $0.508(8)$ & $0.75(3)$ \\
\hline \hline
\end{tabular}


TABLE III. Interfacial free energy anisotropy parameters for the truncated Lennard-Jones at three different temperatures. For reference, the same quantities for the hard-sphere system (Ref. 9) are also included. Numbers in parentheses reflect estimated error in the last digit(s) shown.

\begin{tabular}{|c|c|c|c|c|c|}
\hline & & & & $\underline{\gamma_{100}-\gamma_{110}}$ & $\underline{\gamma_{100}-\gamma_{111}}$ \\
\hline & $\gamma_{0}$ & $\epsilon_{1}$ & $\epsilon_{2}$ & $\gamma_{0}$ & $\gamma_{0}$ \\
\hline Lennard Jones $T^{*}=0.617$ & $0.360(2) \epsilon / \sigma^{2}$ & $0.093(17)$ & $-0.011(4)$ & $0.03(1)$ & $0.07(1)$ \\
\hline$T^{*}=1.0$ & $0.539(4) \epsilon / \sigma^{2}$ & $0.13(3)$ & $-0.022(9)$ & $0.035(15)$ & $0.10(2)$ \\
\hline$T^{*}=1.5$ & $0.808(13) \epsilon / \sigma^{2}$ & $0.15(6)$ & $-0.03(2)$ & $0.025(3)$ & $0.11(3)$ \\
\hline Hard spheres (Ref. 9) & $0.617(6) k T / \sigma^{2}$ & $0.07(3)$ & $-0.044(12)$ & $-0.032(22)$ & $0.065(22)$ \\
\hline
\end{tabular}

The second source of hysteresis is the fluctuation in the interface position at the end of step 4 . When the cleaving potential is removed from the interfacial system in step 4, the position of the interface is no longer tied to the cleaving plane. Because the system contains two interfaces, they can change their position without disturbing system equilibrium by the process of melting at one interface and simultaneous freezing at the other interface. The difficulty in verifying the reversibility of step 4 is that the mobility of the interfaces causes the reverse process to follow a slightly different thermodynamic integration path. To deal with this problem, we try the reverse step 4 process on the interfacial systems after several equilibration runs of various duration and select the one with the path closest to the forward process. The difference in the calculated work during the forward and reverse processes is accounted for in the estimate of the error range given in Table II.

\section{ANALYSIS AND SUMMARY}

The error bars on the calculations described above are small enough to resolve the anisotropy in the interfacial free energy for the Lennard-Jones system. While we have determined $\gamma$ only for the [100], [110], and [111] directions, it is possible to extract from these data some information as to the full angular dependence of the free energy. Defining the orientation unit vector $\hat{\mathbf{n}}$ as the unit vector perpendicular to the interfacial plane, one defines an orientation dependent interfacial free energy $\gamma(\hat{\mathbf{n}})$, which can be parametrized by an expansion in terms of cubic harmonics. One such expansion, due to Fehlner and Vosko, ${ }^{20}$ has been recently applied to the interfacial free energy of $\mathrm{Ni} / \mathrm{Cu}$ alloys by Asta et al. ${ }^{21}$ In terms of the Cartesian components of $\hat{\mathbf{n}}=\left\{n_{1}, n_{2}, n_{3}\right\}$, this expansion (truncated at sixth-order) is

$$
\begin{aligned}
\gamma(\hat{\mathbf{n}})= & \gamma_{0}\left[1+\epsilon_{1}\left(\sum_{i=1}^{3} n_{i}^{4}-\frac{3}{5}\right)\right. \\
& \left.+\epsilon_{2}\left(\sum_{i=1}^{3} n_{i}^{4}+66 n_{1}^{2} n_{2}^{2} n_{3}^{2}-\frac{17}{7}\right)\right],
\end{aligned}
$$

where $\gamma_{0}$ is the orientationally averaged interfacial free energy and $\epsilon_{1}$ and $\epsilon_{2}$ are expansion coefficients. This parametrization has an advantage over the so-called "Kubic Harmonic" expansion ${ }^{22}$ that has been used recently to parametrize free energy anisotropy in metals ${ }^{10,11}$ in that the expansion terms are orthogonal. ${ }^{21}$ For the orientations studied here this expansion becomes

$$
\begin{aligned}
& \gamma_{100}=\gamma_{0}\left[1+\frac{2}{5} \epsilon_{1}+\frac{4}{7} \epsilon_{2}\right], \\
& \gamma_{110}=\gamma_{0}\left[1-\frac{1}{10} \epsilon_{1}-\frac{13}{14} \epsilon_{2}\right], \\
& \gamma_{111}=\gamma_{0}\left[1-\frac{4}{15} \epsilon_{1}+\frac{64}{63} \epsilon_{2}\right] .
\end{aligned}
$$

Using our values for $\gamma$ for the [100], [110], and [111] orientations one can solve for the three parameters $\gamma_{0}, \epsilon_{1}$, and $\epsilon_{2}$ :

$$
\begin{aligned}
& \gamma_{0}=\frac{10 \gamma_{100}+16 \gamma_{110}+9 \gamma_{111}}{35}, \\
& \epsilon_{1}=\frac{35 \gamma_{100}-8 \gamma_{110}-27 \gamma_{111}}{22 \gamma_{0}}, \\
& \epsilon_{2}=\frac{3}{22 \gamma_{0}}\left(\gamma_{100}-4 \gamma_{110}+3 \gamma_{111}\right) .
\end{aligned}
$$

The anisotropy parameters for the Lennard-Jones system studied here, as well as those for the hard-sphere system calculated from the data from our earlier calculation, ${ }^{9}$ are summarized in Table III. Also included in Table III are the values of $\left(\gamma_{100}-\gamma_{110}\right) / \gamma_{0}$ and $\left(\gamma_{100}-\gamma_{111}\right) / \gamma_{0}$, which also serve to quantify the anisotropy. The error bars in $\gamma_{0}$ are smaller than those for the raw interfacial free energies since $\gamma_{0}$ represents a weighted average of similar numbers-a process that decreases statistical error. The anisotropy parameters represent differential quantities involving differences between similar numbers (which magnifies relative error) so the relative error bars for those quantities are larger than in the raw data. This is in contrast to the fluctuation method ${ }^{21}$ where the relative error in the interfacial free energy is larger than in the current study (despite the much larger systems), but the anisotropy parameters are more precisely determined due to the fact that in that method the anisotropy is determined from the anisotropy in the interfacial stiffness, which is far greater than that of the interfacial free energy itself.

From the anisotropy parameters given in Table III, the trend is observed that the anisotropy parameters increase in magnitude with increasing temperature, with $\epsilon_{1}$ becoming more positive and $\epsilon_{2}$ becoming more negative. In comparison with the hard-sphere values, the value of $\epsilon_{2}$ approaches the hard-sphere value as $T$ gets larger, but the value of $\epsilon_{1}$, which is at $T^{*}=0.617$ quite close to the hard-sphere value, diverges away from the hard-sphere value as $T$ increases. Of 
course, in the limit of high temperatures, the Lennard-Jones system approaches an inverse 12th power repulsive potential, not the hard-sphere potential, so one would expect the hightemperature behavior of the anisotropy to approach that of the former potential. This implies that any perturbation theory for the interfacial free energy with a hard-sphere reference potential will not be adequate to predict anisotropy, and that a study of the anisotropy for a variety of different possible repulsive potentials would be very useful.

In recent work, ${ }^{13}$ we have pointed out that the interfacial free energy for simple systems with face-centered cubic (fcc) crystal structures can be quantitatively described by a hardsphere model. It is useful to check this hypothesis here, since the LJ system is a standard molecular model that freezes to a fcc crystal, and since the interfacial free energy was determined in this study directly and not indirectly from nucleation data. The hard-sphere model predicts

$$
\gamma_{0}(\mathrm{HS})=0.617 k T / \sigma^{2} \text {, }
$$

where $\sigma^{2}$ is the hard-sphere diameter. For our Lennard-Jones system we can define an effective (temperature-dependent) hard-sphere diameter using the Barker-Henderson criterion $^{23}$ from liquid-state perturbation theory,

$$
\sigma_{\text {eff }}=\int_{0}^{\infty}\left\{1-\exp \left[-u_{r}(r) / k T\right]\right\} d r,
$$

where $u_{r}(r)$ is the repulsive part of the potential, which we define in the Week-Chandler-Anderson sense ${ }^{23}$ as the full potential truncated (and shifted) to zero beyond the minimum of the attractive well. This procedure yields values of $\sigma_{\text {eff }}$ of $1.032 \sigma, 1.016 \sigma$, and $1.000 \sigma$, for $T^{*}=0.617,1.0$, and 1.5 , respectively. Inserting these values into Eq. (15) yields predicted values of $\gamma_{0}$ (in units of $\epsilon / \sigma^{2}$ ) of $0.36,0.60$, and 0.93 , for $T^{*}=0.617,1.0$, and 1.5 , respectively. The agreement with the values listed for LJ in Table III is excellent at the lower temperature, but overestimates the actual value by several percent at the higher temperatures. This agreement gives more evidence to support the general hypothesis ${ }^{13}$ that the interfacial free energy of close packed systems is largely determined by packing considerations, not energy.

It is interesting to note that the anisotropy parameters $\epsilon_{1}$ and $\epsilon_{2}$ for the truncated LJ potential at the triple point $\left(T^{*}\right.$
$=0.617$ and essentially zero pressure) are identical to those calculated $^{21}$ for $\mathrm{Ni}$ at 1.00 atm (also essentially zero pressure). The fact that the parameters are exactly the same for these two (essentially) zero pressure systems is, given the error bars, probably coincidental; however, the data does show that the anisotropy for $\mathrm{Ni}$ is better modelled by a LJ potential than by a hard-sphere potential. More study is required to determine the exact role that details of the potential play in determining interfacial anisotropy.

\section{ACKNOWLEDGMENTS}

This work was performed using the University of Leicester Mathematical Modelling Center's supercomputer, which was purchased through the EPSRC strategic equipment initiative. In addition, BBL gratefully acknowledges support from the National Science Foundation under Grant No. CHE9970903.

${ }^{1}$ W. A. Tiller, The Science of Crystallization: Microscopic Interfacial Phenomena (Cambridge University Press, New York, 1991).

${ }^{2}$ W. J. Boettinger, S. R. Coriell, A. L. Greer, A. Karma, W. Kurz, M. Rappaz, and R. Trivedi, Acta Mater. 48, 43 (2000)

${ }^{3}$ D. P. Woodruff, The Solid-Liquid Interface (Cambridge University Press, London, 1973).

${ }^{4}$ D. Turnbull, J. Appl. Phys. 21, 1022 (1950).

${ }^{5}$ J. M. Howe, Interfaces in Materials (Wiley, New York, 1997).

${ }^{6}$ M. E. Glicksman and N. B. Singh, J. Cryst. Growth 98, 277 (1989).

${ }^{7}$ M. Muschol, D. Liu, and H. Z. Cummins, Phys. Rev. A 46, 1038 (1992).

${ }^{8}$ J. Q. Broughton and G. H. Gilmer, J. Chem. Phys. 84, 5759 (1986).

${ }^{9}$ R. L. Davidchack and B. B. Laird, Phys. Rev. Lett. 85, 4751 (2000).

${ }^{10}$ J. J. Hoyt, M. Asta, and A. Karma, Phys. Rev. Lett. 86, 5530 (2001).

${ }^{11}$ J. Morris, Phys. Rev. B 66, 144104 (2002).

${ }^{12}$ D. Frenkel and B. Smit, Understanding Molecular Simulation, 2nd ed., (Academic, New York, 2002).

${ }^{13}$ B. B. Laird, J. Chem. Phys. 115, 2887 (2001).

${ }^{14}$ J. Q. Broughton and G. H. Gilmer, J. Chem. Phys. 84, 5749 (1986).

${ }^{15}$ J. Q. Broughton and G. H. Gilmer, Acta Metall. 31, 845 (1983).

${ }^{16}$ D. C. Rappaport, The Art of Molecular Dynamics Simulation (Cambridge University Press, New York, 1995).

${ }^{17}$ J. Q. Broughton and G. H. Gilmer, J. Chem. Phys. 84, 5741 (1986).

${ }^{18}$ R. L. Davidchack and B. B. Laird, J. Chem. Phys. 108, 9452 (1998).

${ }^{19}$ W. C. Swope, H. C. Anderson, P. H. Berens, and K. R. Wilson, J. Chem. Phys. 76, 637 (1982).

${ }^{20}$ W. R. Fehlner and S. H. Vosko, Can. J. Phys. 54, 2159 (1976).

${ }^{21}$ M. Asta, J. J. Hoyt, and A. Karma, Phys. Rev. B 66, 100101(R) (2002).

${ }^{22}$ A. L. Altman and A. P. Cracknell, Rev. Mod. Phys. 37, 19 (1965).

${ }^{23}$ J. P. Hansen and I. R. McDonald, Theory of Simple Liquids, 2nd ed. (Academic, New York, 1986). 